\title{
A parametric kind of the poly-Bernoulli polynomials
}

\author{
Mohd Ghayasuddin ${ }^{\mathrm{a}}$, Sunil K. Sharma ${ }^{\mathrm{b}, *}$, Waseem A. Khan ${ }^{\mathrm{c}}$ \\ ${ }^{a}$ Department of Mathematics, Integral University Campus, Shahjahanpur-242001, India. \\ ${ }^{b}$ College of Computer and Information Sciences, Majmaah University, Majmaah 11952, Saudi Arabia. \\ ${ }^{c}$ Department of Mathematics and Natural Sciences, Prince Mohammad Bin Fahd University, P. O. Box: 1664, Al Khobar 31952, Saudi \\ Arabia.
}

\begin{abstract}
In this paper, we propose a parametric kind of poly-Bernoulli polynomials by defining the two specific generating functions. We also investigate some analytical properties (for example, summation formulae, differential formulae and relations with other well-known polynomials and numbers) for our introduced polynomials in a systematic way. Furthermore, we derive two interesting connections of our parametric poly-Bernoulli polynomials with the familiar Voigt functions.
\end{abstract}

Keywords: Polylogarithmic function, poly-Bernoulli numbers and polynomials, Voigt functions.

2020 MSC: 11B83, 11B68, 33E20, 05A15.

(C)2020 All rights reserved.

\section{Introduction}

Throughout in this paper, let $\mathbb{N}, \mathbb{Z}, \mathbb{R}$ and $\mathbb{R}^{+}$be the sets of natural numbers, integers, real numbers and positive real numbers, respectively, and let

$$
\mathbb{N}_{0}:=\{0,1,2,3, \ldots\}=\mathbb{N} \cup\{0\} .
$$

The Appell polynomials $A_{n}(u)$ are described as follows:

$$
f(t) e^{u t}=\sum_{n=0}^{\infty} A_{n}(u) \frac{t^{n}}{n !},
$$

where $f$ is a formal power series in $t$. These polynomials have found notable applications in several branches of mathematics, physics and engineering. In particular, the most important and useful polynomials of this Appell family (so-called Bernoulli polynomials) are obtained by taking $f(t)=\frac{t}{e^{t}-1}$ in (1.1). Hence the Bernoulli polynomials are defined by (see [24]; see also [28])

$$
\frac{t}{e^{t}-1} e^{u t}=\sum_{n=0}^{\infty} \mathbb{B}_{n}(u) \frac{t^{n}}{n !}, \quad(|t|<2 \pi) .
$$

\footnotetext{
*Corresponding author

Email addresses: ghayas.maths@gmail.com (Mohd Ghayasuddin), s.sharma@mu.edu.sa (Sunil K. Sharma), wkhan1@pmu.edu .sa (Waseem A. Khan)
}

doi: $10.22436 /$ jmcs.021.04.04

Received: 2020-02-18 Revised: 2020-04-11 Accepted: 2020-04-14 
For $u=0,(1.2)$ reduces to

$$
\frac{t}{e^{t}-1}=\sum_{n=0}^{\infty} \mathbb{B}_{n} \frac{t^{n}}{n !}
$$

where $\mathbb{B}_{\mathfrak{n}}$ are the familiar Bernoulli numbers, which are directly connected with several combinatorial numbers such as Cauchy numbers, harmonic numbers and Stirling numbers of the second kind.

Due to notable applications of Bernoulli polynomials, Apostol [1] defined a new generalized Bernoulli polynomials $\mathbb{B}_{\mathfrak{n}}(u ; \beta)$ so-called Apostol-Bernoulli polynomials by introducing a complex parameter $\beta$ in the generating function of classical Bernoulli polynomials as follows:

$$
\frac{t}{\beta e^{t}-1} e^{u t}=\sum_{n=0}^{\infty} \mathbb{B}_{n}(u ; \beta) \frac{t^{n}}{n !}, \quad(\beta \in \mathbb{C},|t|<|\log \beta|) .
$$

For $\beta=1$, (1.4) easily reduces to (1.2). Moreover, we have

$$
\mathbb{B}_{\mathfrak{n}}(\beta)=\mathbb{B}_{\mathfrak{n}}(0 ; \beta),
$$

where $\mathbb{B}_{\mathfrak{n}}(\beta)$ denotes the corresponding Apostol-Bernoulli numbers (see [1], see also [26]).

The higher order Bernoulli polynomials and numbers are given, respectively, by (see [21], [22])

$$
\left(\frac{t}{e^{t}-1}\right)^{p} e^{u t}=\sum_{n=0}^{\infty} B_{n}^{(p)}(u) \frac{t^{n}}{n !}
$$

and

$$
\left(\frac{t}{e^{t}-1}\right)^{p}=\sum_{n=0}^{\infty} \mathbb{B}_{n}^{(p)} \frac{t^{n}}{n !}, \quad\left(|t|<2 \pi ; 1^{p}:=1\right) .
$$

If we set $p=1$ in (1.5) and (1.6) then we get the classical Bernoulli polynomials and numbers defined by (1.2) and (1.3), respectively.

Motivated by the useful extensions of classical Bernoulli polynomials, Luo and Srivastava in [20] introduced and investigated the following Apostol-Bernoulli polynomials $\mathbb{B}_{n}^{(p)}(u ; \beta)$ of order $p$ :

$$
\left(\frac{t}{\beta e^{t}-1}\right)^{p} e^{u t}=\sum_{n=0}^{\infty} \mathbb{B}_{n}^{(p)}(u ; \beta) \frac{t^{n}}{n !}, \quad\left(\beta \in \mathbb{C},|t|<|\log \beta| ; 1^{p}:=1\right) .
$$

A further generalization of Bernoulli polynomials so-called poly-Bernoulli polynomials are defined by Bayad and Hamahata [3] as follows:

$$
\frac{\mathrm{L} i_{k}\left(1-e^{-t}\right)}{1-e^{-t}} e^{u t}=\sum_{n=0}^{\infty} \mathbb{B}_{n}^{(k)}(u) \frac{t^{n}}{n !},
$$

where $L i_{k}$ denotes the polylogarithmic functions defined by

$$
\operatorname{Li}_{k}(z)=\sum_{n=0}^{\infty} \frac{z^{n}}{n^{k}}, \quad(k \in \mathbb{Z}) .
$$

For more details about the above type polynomials and numbers, we refer to [2, 6-23] and the references cited therein.

Recently, by using the exponential as well as trigonometric functions, Kim and Ryoo [17] proposed the two parametric kinds of Bernoulli polynomials as follows:

$$
\left(\frac{t}{e^{t}-1}\right) e^{u t} \cos v t=\sum_{n=0}^{\infty} \mathbb{B}_{n}^{(c)}(u, v) \frac{t^{n}}{n !} \text { and }\left(\frac{t}{e^{t}-1}\right) e^{u t} \sin v t=\sum_{n=0}^{\infty} \mathbb{B}_{n}^{(s)}(u, v) \frac{t^{n}}{n !} \text {. }
$$


Moreover, they also introduced and investigated the two parametric kinds of Euler polynomials in [17].

Afterwards, Srivastava et al. [29] presented the following two parametric kinds of Apostol-Bernoulli polynomials:

$$
\left(\frac{t}{\beta e^{t}-1}\right) e^{u t} \cos v t=\sum_{n=0}^{\infty} \mathbb{B}_{n}^{(c)}(u, v ; \beta) \frac{t^{n}}{n !}
$$

and

$$
\left(\frac{t}{\beta e^{t}-1}\right) e^{u t} \sin v t=\sum_{n=0}^{\infty} \mathbb{B}_{n}^{(s)}(u, v ; \beta) \frac{t^{n}}{n !} .
$$

More recently, Srivastava and Kizilates [27] considered a further generalization of above parametric Apostol-Bernoulli polynomials as follows:

$$
\left(\frac{t}{\beta e^{t}-1}\right)^{p} e^{u t} \cos v t=\sum_{n=0}^{\infty} \mathbb{B}_{n}^{(c, p)}(u, v ; \beta) \frac{t^{n}}{n !}
$$

and

$$
\left(\frac{t}{\beta e^{t}-1}\right)^{p} e^{u t} \sin v t=\sum_{n=0}^{\infty} \mathbb{B}_{n}^{(s, p)}(u, v ; \beta) \frac{t^{n}}{n !} .
$$

It is clear that the case $p=1$ in (1.10) and (1.11), yields the known parametric kinds of Apostol-Bernoulli polynomials defined by (1.8) and (1.9), respectively.

The main object of this paper is to present the two parametric kinds of poly-Bernoulli polynomials by means of the two specific generating functions. Some fundamental properties, such as summation formulae, differential formulae and relations with other polynomials and numbers for our introduced polynomials are also investigated. Furthermore, we derive the connections of our parametric poly-Bernoulli polynomials with the familiar Voigt functions $K(u, v)$ and $L(u, v)$. The Voigt functions $K(u, v)$ and $L(u, v)$ are defined by the following Laplace type integral transforms (see [25]):

$$
\mathrm{K}(\mathrm{u}, v)=\frac{1}{\sqrt{\pi}} \int_{0}^{\infty} e^{-v t-\mathrm{t}^{2} / 4} \cos (u t) d t, \quad\left(u \in \mathbb{R}, v \in \mathbb{R}^{+}\right)
$$

and

$$
\mathrm{L}(\mathrm{u}, \boldsymbol{v})=\frac{1}{\sqrt{\pi}} \int_{0}^{\infty} e^{-v \mathrm{t}-\mathrm{t}^{2} / 4} \sin (u \mathrm{t}) \mathrm{dt}, \quad\left(u \in \mathbb{R}, v \in \mathbb{R}^{+}\right)
$$

\section{Parametric kinds of poly-Bernoulli polynomials}

In this section, we present two parametric kinds of poly-Bernoulli polynomials by defining the two specific generating functions.

By virtue of (1.7), we consider

$$
\sum_{n=0}^{\infty} \mathbb{B}_{n}^{(k)}(u+i v) \frac{t^{n}}{n !}=\frac{L i_{k}\left(1-e^{-t}\right)}{1-e^{-t}} e^{(u+i v) t}=\frac{L i_{k}\left(1-e^{-t}\right)}{1-e^{-t}} e^{u t}[\cos v t+i \sin v t], \quad(u, v \in \mathbb{R})
$$

and

$$
\sum_{n=0}^{\infty} \mathbb{B}_{n}^{(k)}(u-i v) \frac{t^{n}}{n !}=\frac{L i_{k}\left(1-e^{-t}\right)}{1-e^{-t}} e^{u t}[\cos v t-i \sin v t] .
$$

From (2.1) and (2.2), we have

$$
\frac{L i_{k}\left(1-e^{-t}\right)}{1-e^{-t}} e^{u t} \cos v t=\sum_{n=0}^{\infty}\left[\frac{\mathbb{B}_{n}^{(k)}(u+i v)+\mathbb{B}_{n}^{(k)}(u-\mathfrak{i} v)}{2}\right] \frac{t^{n}}{n !}
$$


and

$$
\frac{L i_{k}\left(1-e^{-t}\right)}{1-e^{-t}} e^{u t} \sin v t=\sum_{n=0}^{\infty}\left[\frac{\mathbb{B}_{n}^{(k)}(u+i v)+\mathbb{B}_{n}^{(k)}(u-i v)}{2 i}\right] \frac{t^{n}}{n !} .
$$

Hence, we define here the two parametric kinds (cosine and sine) of poly-Bernoulli polynomials, as follow.

Definition 2.1. Two parametric kinds of poly-Bernoulli polynomials or the cosine-poly-Bernoulli polynomials $\mathbb{B}_{n}^{(c, k)}(u, v)$ and sine-poly-Bernoulli polynomials $\mathbb{B}_{n}^{(s, k)}(u, v)$ for nonnegative integer $n$ are defined, respectively, by

$$
\sum_{n=0}^{\infty} \mathbb{B}_{n}^{(c, k)}(u, v) \frac{t^{n}}{n !}=\frac{L i_{k}\left(1-e^{-t}\right)}{1-e^{-t}} e^{u t} \cos v t, \quad(u, v \in \mathbb{R},|t|<2 \pi)
$$

and

$$
\sum_{n=0}^{\infty} \mathbb{B}_{n}^{(s, k)}(u, v) \frac{t^{n}}{n !}=\frac{L i_{k}\left(1-e^{-t}\right)}{1-e^{-t}} e^{u t} \sin v t, \quad(u, v \in \mathbb{R},|t|<2 \pi) .
$$

It is noticed that

$$
\begin{array}{rlrl}
\mathbb{B}_{n}^{(c, k)}(u, 0) & =\mathbb{B}_{n}^{(c, k)}(u), & \mathbb{B}_{n}^{(s, k)}(u, 0)=0, & \mathbb{B}_{n}^{(c, k)}(0, v)=\mathbb{B}_{n}^{(c, k)}(v), \\
\mathbb{B}_{n}^{(s, k)}(0, v)=\mathbb{B}_{n}^{(s, k)}(v), & \mathbb{B}_{n}^{(c, k)}(0,0)=\mathbb{B}_{n}^{(c, k),} & \mathbb{B}_{n}^{(s, k)}(0,0)=0 .
\end{array}
$$

Moreover, we consider

$$
e^{u t} \cos v t=\sum_{n=0}^{\infty} C_{n}(u, v) \frac{t^{n}}{n !}, \quad \text { and } \quad e^{u t} \sin v t=\sum_{n=0}^{\infty} S_{n}(u, v) \frac{t^{n}}{n !} .
$$

The Taylor-Maclaurin expansions of $e^{u t} \cos v t$ and $e^{u t} \sin v t$ for real $u$ and $v$ are given, respectively, by

$$
e^{u t} \cos v t=\sum_{n=0}^{\infty}\left(\sum_{m=0}^{\left[\frac{n}{2}\right]}\left(\begin{array}{c}
n \\
2 m
\end{array}\right)(-1)^{m} u^{n-2 m} v^{2 m}\right) \frac{t^{n}}{n !}
$$

and

$$
e^{u t} \sin v t=\sum_{n=0}^{\infty}\left(\sum_{m=0}^{\left[\frac{n-1}{2}\right]}\left(\begin{array}{c}
n \\
2 m+1
\end{array}\right)(-1)^{m} u^{n-2 m-1} v^{2 m+1}\right) \frac{t^{n}}{n !},
$$

where [.] denotes the greatest integer.

Hence, from (2.4)-(2.6), we have

$$
C_{n}(u, v)=\sum_{m=0}^{\left[\frac{n}{2}\right]}\left(\begin{array}{c}
n \\
2 m
\end{array}\right)(-1)^{m} u^{n-2 m} v^{2 m} \quad \text { and } \quad S_{n}(u, v)=\sum_{m=0}^{\left[\frac{n-1}{2}\right]}\left(\begin{array}{c}
n \\
2 m+1
\end{array}\right)(-1)^{m} u^{n-2 m-1} v^{2 m+1} \text {. }
$$

\section{Properties of $\mathbb{B}_{\mathfrak{n}}^{(c, k)}(u, v)$ and $\mathbb{B}_{n}^{(s, k)}(u, v)$}

In this section, we derive some fundamental properties (like summation formulae, differential formulae and relations with other standard polynomials and numbers) of our two parametric kinds polyBernoulli polynomials. 
Theorem 3.1. For $n \in \mathbb{N}_{0}$, each of the following identities holds true:

$$
\mathbb{B}_{n}^{(c, k)}(u, v)=\sum_{r=0}^{n}\left(\begin{array}{l}
n \\
r
\end{array}\right) \mathbb{B}_{n-r}^{(k)} C_{r}(u, v)
$$

and

$$
\mathbb{B}_{n}^{(s, k)}(u, v)=\sum_{r=0}^{n}\left(\begin{array}{l}
n \\
r
\end{array}\right) \mathbb{B}_{n-r}^{(k)} S_{r}(u, v)
$$

Proof. Taking

$$
\begin{aligned}
\frac{L i_{k}\left(1-e^{-t}\right)}{1-e^{-t}} e^{u t} \cos v t & =\sum_{n=0}^{\infty} \mathbb{B}_{n}^{(k)} \frac{t^{n}}{n !} \sum_{r=0}^{\infty} C_{r}(u, v) \frac{t^{r}}{r !} \\
\sum_{n=0}^{\infty} \mathbb{B}_{n}^{(c, k)}(u, v) \frac{t^{n}}{n !} & =\sum_{n=0}^{\infty} \sum_{r=0}^{\infty} \mathbb{B}_{n}^{(k)} C_{r}(u, v) \frac{t^{n+r}}{n ! r !}=\sum_{n=0}^{\infty} \sum_{r=0}^{n} \mathbb{B}_{n-r}^{(k)} C_{r}(u, v) \frac{t^{n}}{(n-r) ! r !}
\end{aligned}
$$

which on equating the like powers of $t$ gives our needed identity (3.1). Similarly, we can prove our second identity (3.2).

Theorem 3.2. For $\mathrm{n} \in \mathbb{N}_{0}$ and $w \in \mathbb{R}$, we have

$$
\mathbb{B}_{n}^{(c, k)}(u+w, v)=\sum_{r=0}^{n}\left(\begin{array}{l}
n \\
r
\end{array}\right) w^{r} \mathbb{B}_{n-r}^{(c, k)}(u, v)
$$

and

$$
\mathbb{B}_{n}^{(s, k)}(u+w, v)=\sum_{r=0}^{n}\left(\begin{array}{l}
n \\
r
\end{array}\right) w^{r} \mathbb{B}_{n-r}^{(s, k)}(u, v)
$$

Proof. From (2.3), we get

$$
\begin{aligned}
\sum_{n=0}^{\infty} \mathbb{B}_{n}^{(c, k)}(u+w, v) \frac{t^{n}}{n !} & =\frac{L i_{k}\left(1-e^{-t}\right)}{1-e^{-t}} e^{(u+w) t} \cos v t \\
& =\frac{L i_{k}\left(1-e^{-t}\right)}{1-e^{-t}} e^{u t} \cos v t e^{w t} \\
& =\sum_{n=0}^{\infty} \mathbb{B}_{n}^{(c, k)}(u, v) \frac{t^{n}}{n !} \sum_{r=0}^{\infty} \frac{(w t)^{r}}{r !}=\sum_{n=0}^{\infty} \sum_{r=0}^{n}\left(\begin{array}{l}
n \\
r
\end{array}\right) w^{r} \mathbb{B}_{n-r}^{(c, k)}(u, v) \frac{t^{n}}{n !},
\end{aligned}
$$

which on equating the coefficients of $\frac{t^{n}}{n !}$, yields our claimed result (3.3). Similarly, we can derive our second result (3.4).

Corollary 3.3. On setting $w=u$ in Theorem 3.2, we have

$$
\mathbb{B}_{n}^{(c, k)}(2 u, v)=\sum_{r=0}^{n}\left(\begin{array}{l}
n \\
r
\end{array}\right) u^{r} \mathbb{B}_{n-r}^{(c, k)}(u, v) \quad \text { and } \quad \mathbb{B}_{n}^{(s, k)}(2 u, v)=\sum_{r=0}^{n}\left(\begin{array}{l}
n \\
r
\end{array}\right) u^{r} \mathbb{B}_{n-r}^{(s, k)}(u, v) \text {. }
$$

Corollary 3.4. On setting $w=1$ in Theorem 3.2 , we have

$$
\mathbb{B}_{n}^{(c, k)}(u+1, v)=\sum_{r=0}^{n}\left(\begin{array}{c}
n \\
r
\end{array}\right) \mathbb{B}_{n-r}^{(c, k)}(u, v) \quad \text { and } \quad \mathbb{B}_{n}^{(s, k)}(u+1, v)=\sum_{r=0}^{n}\left(\begin{array}{c}
n \\
r
\end{array}\right) \mathbb{B}_{n-r}^{(s, k)}(u, v) .
$$


Theorem 3.5. For $\mathrm{n} \in \mathbb{N}_{0}$ and $w \in \mathbb{R}$, we have

$$
\mathbb{B}_{n}^{(c, k)}(u, v+w)=\sum_{m=0}^{\left[\frac{n}{2}\right]}\left(\begin{array}{c}
n \\
2 m
\end{array}\right)(-1)^{m} w^{2 m} \mathbb{B}_{n-2 m}^{(c, k)}(u, v)-\sum_{m=0}^{\left[\frac{n-1}{2}\right]} \frac{(-1)^{m} n ! w^{2 m+1} \mathbb{B}_{n-2 m-1}^{(s, k)}(u, v)}{m !(n-2 m-1) ! 2^{2 m}\left(\frac{3}{2}\right)_{m}}
$$

and

$$
\mathbb{B}_{n}^{(s, k)}(u, v+w)=\sum_{m=0}^{\left[\frac{n}{2}\right]}\left(\begin{array}{c}
n \\
2 m
\end{array}\right)(-1)^{m} w^{2 m} \mathbb{B}_{n-2 m}^{(s, k)}(u, v)+\sum_{m=0}^{\left[\frac{n-1}{2}\right]} \frac{(-1)^{m} n ! w^{2 m+1} \mathbb{B}_{n-2 m-1}^{(c, k)}(u, v)}{m !(n-2 m-1) ! 2^{2 m}\left(\frac{3}{2}\right)_{m}} .
$$

Proof. We have

$$
\begin{aligned}
\sum_{n=0}^{\infty} \mathbb{B}_{n}^{(c, k)}(u, v+w) \frac{t^{n}}{n !} \\
=\frac{L i_{k}\left(1-e^{-t}\right)}{1-e^{-t}} e^{u t} \cos (v+w) t \\
=\frac{L i_{k}\left(1-e^{-t}\right)}{1-e^{-t}} e^{u t}[\cos v t \cos w t-\sin v t \sin w t] \\
=\frac{L i_{k}\left(1-e^{-t}\right)}{1-e^{-t}} e^{u t} \cos v t \sum_{m=0}^{\infty} \frac{(-1)^{m}(w t)^{2 m}}{2 m !}-\frac{L i_{k}\left(1-e^{-t}\right)}{1-e^{-t}} e^{u t} \sin v t \sum_{m=0}^{\infty} \frac{(-1)^{m}(w t)^{2 m+1}}{2^{2 m} m !\left(\frac{3}{2}\right)_{m}} \\
=\sum_{n=0}^{\infty} \mathbb{B}_{n}^{(c, k)}(u, v) \frac{t^{n}}{n !} \sum_{m=0}^{\infty} \frac{(-1)^{m}(w t)^{2 m}}{2 m !}-\sum_{n=0}^{\infty} \mathbb{B}_{n}^{(s, k)}(u, v) \frac{t^{n}}{n !} \sum_{m=0}^{\infty} \frac{(-1)^{m}(w t)^{2 m+1}}{2^{2 m} m !\left(\frac{3}{2}\right)_{m}} \\
=\sum_{n=0}^{\infty} \sum_{m=0}^{\infty} \frac{(-1)^{m}(w)^{2 m} \mathbb{B}_{n}^{(c, k)}(u, v) t^{n+2 m}}{2 m ! n !}-\sum_{n=0}^{\infty} \sum_{m=0}^{\infty} \frac{(-1)^{m} w^{2 m+1} \mathbb{B}_{n}^{(s, k)}(u, v) t^{n+2 m+1}}{2^{2 m} m ! n !\left(\frac{3}{2}\right)_{m}} \\
=\sum_{n=0}^{\infty} \sum_{m=0}^{\left[\frac{n}{2}\right]} \frac{(-1)^{m}(w)^{2 m} \mathbb{B}_{n-2 m}^{(c, k)}(u, v) t^{n}}{2 m !(n-2 m) !}-\sum_{n=0}^{\infty} \sum_{m=0}^{\left[\frac{n-1}{2}\right]} \frac{(-1)^{m} w^{2 m+1} \mathbb{B}_{n-2 m-1}^{(s, k)}(u, v) t^{n}}{2^{2 m} m !(n-2 m-1) !\left(\frac{3}{2}\right)_{m}}
\end{aligned}
$$

Finally, by comparing the coefficients of $t^{n}$, we get our assertion (3.5). In a similar way, we can derive our second assertion (3.5).

Corollary 3.6. If we replace $w$ by $-w$ in Theorem 3.5 , then we have

$$
\mathbb{B}_{n}^{(c, k)}(u, v-w)=\sum_{m=0}^{\left[\frac{n}{2}\right]}\left(\begin{array}{c}
n \\
2 m
\end{array}\right)(-1)^{m} w^{2 m} \mathbb{B}_{n-2 m}^{(c, k)}(u, v)+\sum_{m=0}^{\left[\frac{n-1}{2}\right]} \frac{(-1)^{m} n ! w^{2 m+1} \mathbb{B}_{n-2 m-1}^{(s, k)}(u, v)}{m !(n-2 m-1) ! 2^{2 m}\left(\frac{3}{2}\right)_{m}}
$$

and

$$
\mathbb{B}_{n}^{(s, k)}(u, v-w)=\sum_{m=0}^{\left[\frac{n}{2}\right]}\left(\begin{array}{c}
n \\
2 m
\end{array}\right)(-1)^{m} w^{2 m} \mathbb{B}_{n-2 m}^{(s, k)}(u, v)-\sum_{m=0}^{\left[\frac{n-1}{2}\right]} \frac{(-1)^{m} n ! w^{2 m+1} \mathbb{B}_{n-2 m-1}^{(c, k)}(u, v)}{m !(n-2 m-1) ! 2^{2 m}\left(\frac{3}{2}\right)_{m}}
$$

Theorem 3.7. Let $\mathrm{n} \in \mathbb{N}$. Then we have

$$
\begin{aligned}
\frac{\partial}{\partial u} \mathbb{B}_{n}^{(c, k)}(u, v) & =n \mathbb{B}_{n-1}^{(c, k)}(u, v), \\
\frac{\partial}{\partial v} \mathbb{B}_{n}^{(c, k)}(u, v) & =-n \mathbb{B}_{n-1}^{(s, k)}(u, v),
\end{aligned}
$$




$$
\frac{\partial}{\partial u} \mathbb{B}_{n}^{(s, k)}(u, v)=n \mathbb{B}_{n-1}^{(s, k)}(u, v),
$$

and

$$
\frac{\partial}{\partial v} \mathbb{B}_{n}^{(s, k)}(u, v)=n \mathbb{B}_{n-1}^{(c, k)}(u, v)
$$

Proof. From (2.3), we have

$$
\sum_{n=0}^{\infty} \mathbb{B}_{n}^{(c, k)}(u, v) \frac{t^{n}}{n !}=\frac{L i_{k}\left(1-e^{-t}\right)}{1-e^{-t}} e^{u t} \cos v t
$$

On partially differentiating with respect to $u$, we get

$$
\sum_{n=0}^{\infty} \frac{\partial}{\partial u} \mathbb{B}_{n}^{(c, k)}(u, v) \frac{t^{n}}{n !}=t \frac{L i_{k}\left(1-e^{-t}\right)}{1-e^{-t}} e^{u t} \cos v t=\sum_{n=0}^{\infty} \mathbb{B}_{n}^{(c, k)}(u, v) \frac{t^{n+1}}{n !}
$$

which, upon comparing the coefficients of $t^{n}$ on both sides, yields the desired result (3.5). Similarly, we can derive (3.6)-(3.8).

The rasing factorial, denoted by $(u)^{\gamma}$, is defined by

$$
(u)^{\gamma}=u(u+1) \cdots(u+\gamma-1) .
$$

We also note that the negative binomial expansion is given by

$$
(\beta+\gamma)^{-k}=\sum_{m=0}^{\infty}(-1)^{m}\left(\begin{array}{c}
k+m-1 \\
m
\end{array}\right) \beta^{m} \gamma^{-k-m} .
$$

Theorem 3.8. For $n, m \in \mathbb{N}_{0}$, each of the following identities holds true:

$$
\mathbb{B}_{n}^{(c, k)}(u, v)=\sum_{m=0}^{\infty} \sum_{l=0}^{n}\left(\begin{array}{l}
n \\
l
\end{array}\right)(u)^{(m)} S_{2}(l, m) B_{n-l}^{(c, k)}(-m, v)
$$

and

$$
\mathbb{B}_{n}^{(s, k)}(u, v)=\sum_{m=0}^{\infty} \sum_{l=0}^{n}\left(\begin{array}{l}
n \\
l
\end{array}\right)(u)^{(m)} S_{2}(l, m) \mathbb{B}_{n-l}^{(s, k)}(-m, v),
$$

where $S_{2}(l, m)$ denotes the Stirling numbers of second kind and is defined by (see [4])

$$
\sum_{l=0}^{\infty} S_{2}(l, m) \frac{t^{l}}{l !}=\frac{\left(e^{t}-1\right)^{m}}{m !}
$$

Proof. From (2.3), we have

$$
\begin{aligned}
\sum_{n=0}^{\infty} \mathbb{B}_{n}^{(c, k)}(u, v) \frac{t^{n}}{n !} & =\frac{L i_{k}\left(1-e^{-t}\right)}{1-e^{-t}} e^{u t} \cos v t \\
& =\frac{L i_{k}\left(1-e^{-t}\right)}{1-e^{-t}}\left[1-\left(1-e^{-t}\right)\right]^{-u} \cos v t \\
& =\frac{L i_{k}\left(1-e^{-t}\right)}{1-e^{-t}} \cos v t \sum_{m=0}^{\infty}\left(\begin{array}{c}
u+m-1 \\
m
\end{array}\right)\left(1-e^{-t}\right)^{m}
\end{aligned}
$$




$$
\begin{aligned}
& =\sum_{m=0}^{\infty}(u)^{(m)} \frac{\left(e^{t}-1\right)^{m}}{m !} \frac{L i_{k}\left(1-e^{-t}\right)}{1-e^{-t}} e^{-m t} \cos v t \\
& =\sum_{m=0}^{\infty}(u)^{(m)} \sum_{l=0}^{\infty} S_{2}(l, m) \frac{t^{l}}{l !} \sum_{n=0}^{\infty} \mathbb{B}_{n}^{(c, k)}(-m, v) \frac{t^{n}}{n !} \\
& =\sum_{n=0}^{\infty} \sum_{m=0}^{\infty} \sum_{l=0}^{n}\left(\begin{array}{l}
n \\
l
\end{array}\right)(u)^{m} S_{2}(l, m) B_{n-l}^{(c, k)}(-m, v) \frac{t^{n}}{n !}
\end{aligned}
$$

which gives our needed result (3.9). Similarly, we can prove our second relation (3.10).

Theorem 3.9. For $\mathrm{n} \in \mathbb{N}$, we have

$$
\mathbb{B}_{n}^{(c, k)}(u+1, v)-\mathbb{B}_{n}^{(c, k)}(u, v)=\sum_{m=0}^{n-1} \frac{n !}{m !(n-1-m) !} \mathbb{B}_{n-1-m}^{(c, k)}(u, v) \mathbb{B}_{m}^{(-1)}
$$

and

$$
\mathbb{B}_{n}^{(s, k)}(u+1, v)-\mathbb{B}_{n}^{(s, k)}(u, v)=\sum_{m=0}^{n-1} \frac{n !}{m !(n-1-m) !} \mathbb{B}_{n-1-m}^{(s, k)}(u, v) \mathbb{B}_{m}^{(-1)},
$$

where $\mathbb{B}_{\mathrm{m}}^{(-1)}$ are the generalized Bernoulli numbers given in (1.6).

Proof. Taking

$$
\begin{aligned}
\frac{L i_{k}\left(1-e^{-t}\right)}{1-e^{-t}} e^{u t} \cos v t\left(e^{t}-1\right) & =t \frac{\operatorname{Li} k\left(1-e^{-t}\right)}{1-e^{-t}} e^{u t} \cos v t\left(\frac{t}{e^{t}-1}\right)^{-1} \\
& =t \sum_{n=0}^{\infty} \mathbb{B}_{n}^{(c, k)}(u, v) \frac{t^{n}}{n !} \sum_{m=0}^{\infty} \mathbb{B}_{m}^{(-1)} \frac{t^{m}}{m !}, \\
\sum_{n=0}^{\infty} \mathbb{B}_{n}^{(c, k)}(u+1, v) \frac{t^{n}}{n !}-\sum_{n=0}^{\infty} \mathbb{B}_{n}^{(c, k)}(u, v) \frac{t^{n}}{n !} & =\sum_{n=0}^{\infty} \sum_{m=0}^{\infty} \mathbb{B}_{n}^{(c, k)}(u, v) \mathbb{B}_{m}^{(-1)} \frac{t^{n+m+1}}{n ! m !} \\
& =\sum_{n=0}^{\infty} \sum_{m=0}^{n} \mathbb{B}_{n-m}^{(c, k)}(u, v) \mathbb{B}_{m}^{(-1)} \frac{t^{n+1}}{(n-m) ! m !} .
\end{aligned}
$$

By comparing the coefficients of $t^{n}$ on both sides, we get our claimed result (3.11). In a similar way, we can derive our second result (3.12).

Theorem 3.10. For $n, m \in \mathbb{N}_{0}$, each of the following relations holds true:

$$
\mathbb{B}_{n}^{(c, k)}(u+1, v)+\mathbb{B}_{n}^{(c, k)}(u, v)=2 \sum_{m=0}^{n}\left(\begin{array}{c}
n \\
m
\end{array}\right) \mathbb{B}_{n-m}^{(c, k)}(u, v) \mathbb{E}_{m}^{(-1)}
$$

and

$$
\mathbb{B}_{n}^{(s, k)}(u+1, v)+\mathbb{B}_{n}^{(s, k)}(u, v)=2 \sum_{m=0}^{n}\left(\begin{array}{l}
n \\
m
\end{array}\right) \mathbb{B}_{n-m}^{(s, k)}(u, v) \mathbb{E}_{m}^{(-1)}
$$

where $\mathbb{E}_{m}^{(-1)}$ are the generalized Euler numbers defined by (see [6])

$$
\left(\frac{2}{e^{t}+1}\right)^{\lambda}=\sum_{m=0}^{\infty} \mathbb{E}_{m}^{(\lambda)} \frac{t^{m}}{m !}
$$


Proof. Taking

$$
\begin{aligned}
\frac{L i_{k}\left(1-e^{-t}\right)}{1-e^{-t}} e^{u t} \cos v t\left(e^{t}+1\right) & =2 \frac{L i_{k}\left(1-e^{-t}\right)}{1-e^{-t}} e^{u t} \cos v t\left(\frac{2}{e^{t}+1}\right)^{-1} \\
& =2 \sum_{n=0}^{\infty} \mathbb{B}_{n}^{(c, k)}(u, v) \frac{t^{n}}{n !} \sum_{m=0}^{\infty} \mathbb{E}_{m}^{(-1)} \frac{t^{m}}{m !}, \\
\sum_{n=0}^{\infty} \mathbb{B}_{n}^{(c, k)}(u+1, v) \frac{t^{n}}{n !}+\sum_{n=0}^{\infty} \mathbb{B}_{n}^{(c, k)}(u, v) \frac{t^{n}}{n !} & =2 \sum_{n=0}^{\infty} \sum_{m=0}^{n} \mathbb{B}_{n-m}^{(c, k)}(u, v) \mathbb{E}_{m}^{(-1)} \frac{t^{n}}{(n-m) ! m !},
\end{aligned}
$$

which, on equating the like powers of $t$ on both sides, yields the desired result (3.13). In a similar way, the assertion (3.14) can be derived.

Theorem 3.11. Let $\mathrm{n} \in \mathbb{N}$. Then we have

$$
\mathbb{B}_{n}^{(c, k)}(u+1, v)+\mathbb{B}_{n}^{(c, k)}(u, v)=2 \sum_{m=0}^{n-1} \frac{n !}{m !(n-1-m) !} \mathbb{B}_{n-1-m}^{(c, k)}(u, v) G_{m}^{(-1)}
$$

and

$$
\mathbb{B}_{n}^{(s, k)}(u+1, v)+\mathbb{B}_{n}^{(s, k)}(u, v)=2 \sum_{m=0}^{n-1} \frac{n !}{m !(n-1-m) !} \mathbb{B}_{n-1-m}^{(s, k)}(u, v) G_{m}^{(-1)},
$$

where $\mathrm{G}_{\mathrm{m}}^{(-1)}$ are the generalized Genocchi numbers defined by (see [6])

$$
\left(\frac{2 t}{e^{t}+1}\right)^{\lambda}=\sum_{m=0}^{\infty} G_{m}^{(\lambda)} \frac{t^{m}}{m !} .
$$

Proof. Proof of this theorem is similar to that of Theorems 3.9 and 3.10.

Theorem 3.12. For $n, m \in \mathbb{N}_{0}$, each of the following relations holds true:

$$
\mathbb{B}_{\mathfrak{n}}^{(c, k)}(u, v)=\sum_{m=0}^{n}\left(\begin{array}{c}
n \\
m
\end{array}\right) \mathbb{B}_{\mathfrak{n}-\mathrm{m}}^{(\mathrm{c}, \mathrm{k})}(v)\left[(1+v) \mathbb{F}_{\mathfrak{m}}(u, v)-v \mathbb{F}_{\mathfrak{m}}(u+1, v)\right]
$$

and

$$
\mathbb{B}_{n}^{(s, k)}(u, v)=\sum_{m=0}^{n}\left(\begin{array}{c}
n \\
m
\end{array}\right) \mathbb{B}_{n-m}^{(s, k)}(v)\left[(1+v) \mathbb{F}_{m}(u, v)-v \mathbb{F}_{m}(u+1, v)\right],
$$

where $\mathbb{F}_{m}(u, v)$ are the classical two-variable Fubini polynomials defined by (see [4])

$$
\sum_{m=0}^{\infty} \mathbb{F}_{m}(u, v) \frac{t^{m}}{m !}=\frac{e^{u t}}{1-v\left(e^{t}-1\right)}
$$

Proof. From (2.3), we have

$$
\begin{aligned}
\sum_{n=0}^{\infty} \mathbb{B}_{n}^{(c, k)}(u, v) \frac{t^{n}}{n !} & =\frac{L i_{k}\left(1-e^{-t}\right)}{1-e^{-t}} e^{u t} \cos v t \\
& =\frac{\operatorname{Li} k\left(1-e^{-t}\right)}{1-e^{-t}} \cos v t \frac{e^{u t}}{\left[1-v\left(e^{t}-1\right)\right]}\left[1-v\left(e^{t}-1\right)\right] \\
& =(1+v) \frac{\operatorname{Li} i_{k}\left(1-e^{-t}\right)}{1-e^{-t}} \cos v t \frac{e^{u t}}{\left[1-v\left(e^{t}-1\right)\right]}-v \frac{L i_{k}\left(1-e^{-t}\right)}{1-e^{-t}} \cos v t \frac{e^{(u+1) t}}{\left[1-v\left(e^{t}-1\right)\right]}
\end{aligned}
$$




$$
\begin{aligned}
& =(1+v) \sum_{n=0}^{\infty} \mathbb{B}_{n}^{(c, k)}(v) \frac{t^{n}}{n !} \sum_{m=0}^{\infty} \mathbb{F}_{m}(u, v) \frac{t^{m}}{m !}-v \sum_{n=0}^{\infty} \mathbb{B}_{n}^{(c, k)}(v) \frac{t^{n}}{n !} \sum_{m=0}^{\infty} \mathbb{F}_{m}(u+1, v) \frac{t^{m}}{m !} \\
& =(1+v) \sum_{n=0}^{\infty} \sum_{m=0}^{n}\left(\begin{array}{c}
n \\
m
\end{array}\right) \mathbb{B}_{n-m}^{(c, k)}(v) \mathbb{F}_{m}(u, v) \frac{t^{n}}{n !}-v \sum_{n=0}^{\infty} \sum_{m=0}^{n}\left(\begin{array}{c}
n \\
m
\end{array}\right) \mathbb{B}_{n-m}^{(c, k)}(v) \mathbb{F}_{m}(u+1, v) \frac{t^{n}}{n !},
\end{aligned}
$$

which, on equating the coefficients of $\frac{t^{n}}{n !}$ on both sides, gives our first claimed result (3.15). Similarly, we can derive our second result (3.16).

Theorem 3.13. For $n, m \in \mathbb{N}_{0}$, each of the following relations holds true:

$$
\mathbb{B}_{n}^{(c, k)}(u, v)=\frac{n ! m !}{2(n+m) !} \sum_{r=0}^{n+m}\left(\begin{array}{c}
n+m \\
r
\end{array}\right) \mathbb{B}_{n+m-r}^{(c, k)}(v) \mathbb{E}_{m, r}(u)+\frac{n ! m !}{2} \sum_{r=0}^{n} \sum_{j=0}^{n} \frac{\mathbb{B}_{n-r-j}^{(c, k)}(v) \mathbb{E}_{m, r}(u)}{r !(n-r-j) !(j+m) !}
$$

and

$$
\mathbb{B}_{n}^{(s, k)}(u, v)=\frac{n ! m !}{2(n+m) !} \sum_{r=0}^{n+m}\left(\begin{array}{c}
n+m \\
r
\end{array}\right) \mathbb{B}_{n+m-r}^{(s, k)}(v) \mathbb{E}_{m, r}(u)+\frac{n ! m !}{2} \sum_{r=0}^{n} \sum_{j=0}^{n} \frac{\mathbb{B}_{n-r-j}^{(s, k)}(v) \mathbb{E}_{m, r}(u)}{r !(n-r-j) !(j+m) !}
$$

where $\mathbb{E}_{\mathrm{m}, \mathrm{r}}(\mathrm{u})$ denotes the truncated Euler polynomials defined by (see [18])

$$
\sum_{r=0}^{\infty} \mathbb{E}_{m, r}(u) \frac{t^{r}}{r !}=\frac{2 t^{m} / m !}{e^{t}+1-\sum_{j=0}^{m-1} t^{j} / j !} e^{u t}
$$

Proof. We have

$$
\begin{aligned}
\sum_{n=0}^{\infty} \mathbb{B}_{n}^{(c, k)}(u, v) \frac{t^{n}}{n !}= & \frac{L i_{k}\left(1-e^{-t}\right)}{1-e^{-t}} e^{u t} \cos v t \frac{2 t^{m} / m !}{\left(e^{t}+1-\sum_{j=0}^{m-1} t^{j} / j !\right)} \frac{\left(e^{t}+1-\sum_{j=0}^{m-1} t^{j} / j !\right)}{2 t^{m} / m !} \\
= & \frac{m !}{2 t^{m}} \sum_{n=0}^{\infty} \mathbb{B}_{n}^{(c, k)}(v) \frac{t^{n}}{n !} \sum_{r=0}^{\infty} \mathbb{E}_{m, r}(u) \frac{t^{r}}{r !}\left(\sum_{j=m}^{\infty} \frac{t^{j}}{j !}+1\right) \\
= & \frac{m !}{2} \sum_{n=0}^{\infty} \sum_{r=0}^{n} \mathbb{B}_{n-r}^{(c, k)}(v) \mathbb{E}_{m, r}(u) \frac{t^{n-m}}{r !(n-r) !}\left(\sum_{j=0}^{\infty} \frac{t^{j+m}}{(j+m) !}+1\right) \\
= & \frac{m !}{2} \sum_{n=0}^{\infty} \sum_{r=0}^{n} \mathbb{B}_{n-r}^{(c, k)}(v) \mathbb{E}_{m, r}(u) \frac{t^{n-m}}{r !(n-r) !} \\
& +\frac{m !}{2} \sum_{n=0}^{\infty} \sum_{r=0}^{n} \sum_{j=0}^{n} \mathbb{B}_{n-r-j}^{(c, k)}(v) \mathbb{E}_{m, r}(u) \frac{t^{n}}{r !(n-r-j) !(j+m) !} .
\end{aligned}
$$

By comparing the coefficients of $t^{n}$ on both sides in the above expression, we easily get our claimed result (3.17). Similarly, we can derive our second result (3.18).

Theorem 3.14. For $\mathrm{n}, \mathrm{m} \in \mathbb{N}_{0}$, we have

$$
\mathbb{B}_{n}^{(c, k)}(u, v)=n ! m ! \sum_{r=0}^{n} \sum_{j=0}^{n} \frac{\mathbb{B}_{n-j-r}^{(c, k)}(v) \mathbb{B}_{m, r}(u)}{r !(n-j-r) !(j+m) !}
$$


and

$$
\mathbb{B}_{n}^{(s, k)}(u, v)=n ! m ! \sum_{r=0}^{n} \sum_{j=0}^{n} \frac{\mathbb{B}_{n-j-r}^{(s, k)}(v) \mathbb{B}_{m, r}(u)}{r !(n-j-r) !(j+m) !},
$$

where $\mathbb{B}_{\mathfrak{m}, \mathrm{r}}(\mathfrak{u})$ denotes the truncated Bernoulli polynomials defined by (see [5])

$$
\sum_{r=0}^{\infty} \mathbb{B}_{m, r}(u) \frac{t^{r}}{r !}=\frac{t^{m} / m !}{\left(e^{t}-\sum_{j=0}^{m-1} t^{j} / j !\right)}
$$

Proof. From (2.3), we have

$$
\begin{aligned}
\sum_{n=0}^{\infty} \mathbb{B}_{n}^{(c, k)}(u, v) \frac{t^{n}}{n !} & =\frac{L i_{k}\left(1-e^{-t}\right)}{1-e^{-t}} e^{u t} \cos v t \frac{t^{m} / m !}{\left(e^{t}-\sum_{j=0}^{m-1} t^{j} / j !\right)} \frac{\left(e^{t}-\sum_{j=0}^{m-1} t^{j} / j !\right)}{t^{m} / m !} \\
& =\frac{m !}{t^{m}} \sum_{n=0}^{\infty} \mathbb{B}_{n}^{(c, k)}(v) \frac{t^{n}}{n !} \sum_{r=0}^{\infty} \mathbb{B}_{m, r}(u) \frac{t^{r}}{r !}\left(\sum_{j=m}^{\infty} \frac{t^{j}}{j !}\right) \\
& =\frac{m !}{t^{m}} \sum_{n=0}^{\infty} \sum_{r=0}^{n} \mathbb{B}_{n-r}^{(c, k)}(v) \mathbb{B}_{m, r}(u) \frac{t^{n}}{r !(n-r) !} \sum_{j=0}^{\infty} \frac{t^{j+m}}{(j+m) !} \\
& =m ! \sum_{n=0}^{\infty} \sum_{r=0}^{n} \sum_{j=0}^{n} \mathbb{B}_{n-j-r}^{(c, k)}(v) \mathbb{B}_{m, r}(u) \frac{t^{n}}{r !(n-j-r) !(j+m) !}
\end{aligned}
$$

which, upon comparing the coefficients of $t^{n}$, yields our needed result (3.19). Similarly, we can establish our second results (3.20).

\section{Connections with Voigt functions}

In this section, we establish the following connections of our introduced polynomials with the well known Voigt functions $\mathrm{K}(\mathrm{u}, v)$ and $\mathrm{L}(\mathrm{u}, \boldsymbol{v})$.

Theorem 4.1. For $n \in \mathbb{N}_{0}$ and $u, v \in \mathbb{R}$, each of the following connections holds true:

$$
\sum_{n=0}^{\infty} \frac{2^{n} \Gamma\left(\frac{n+1}{2}\right) \mathbb{B}_{n}^{(c, k)}(-u, v)}{n !}=\sqrt{\pi} \sum_{n=1}^{\infty} \sum_{r=0}^{n-1}\left(\begin{array}{c}
n-1 \\
r
\end{array}\right) \frac{(-1)^{r}}{n^{k}} K(v, u+r)
$$

and

$$
\sum_{n=0}^{\infty} \frac{2^{n} \Gamma\left(\frac{n+1}{2}\right) \mathbb{B}_{n}^{(s, k)}(-u, v)}{n !}=\sqrt{\pi} \sum_{n=1}^{\infty} \sum_{r=0}^{n-1}\left(\begin{array}{c}
n-1 \\
r
\end{array}\right) \frac{(-1)^{r}}{n^{k}} L(v, u+r) .
$$

Proof. By virtue of (2.3), we have

$$
\begin{aligned}
\sum_{n=0}^{\infty} \mathbb{B}_{n}^{(c, k)}(-u, v) \frac{t^{n}}{n !} & =\frac{L i_{k}\left(1-e^{-t}\right)}{1-e^{-t}} e^{-u t} \cos v t \\
& =\operatorname{Li} i_{k}\left(1-e^{-t}\right)\left(1-e^{-t}\right)^{-1} e^{-u t} \cos v t \\
& =\sum_{n=1}^{\infty} \frac{\left(1-e^{-t}\right)^{n-1}}{n^{k}} e^{-u t} \cos v t=\sum_{n=1}^{\infty} \frac{1}{n^{k}} \sum_{r=0}^{n-1}(-1)^{r}\left(\begin{array}{c}
n-1 \\
r
\end{array}\right) e^{-r t} e^{-u t} \cos v t .
\end{aligned}
$$


Multiplying by $e^{-t^{2} / 4}$ on both sides of last expression and integrating with respect to $t$ from 0 to $\infty$ (after interchanging the order of integration and summations which is permissible due to the uniform convergence), and by using (1.12), we get

$$
\sum_{n=0}^{\infty} \frac{\mathbb{B}_{n}^{(c, k)}(-u, v)}{n !} \int_{0}^{\infty} e^{-\frac{t^{2}}{4}} t^{n} d t=\sqrt{\pi} \sum_{n=1}^{\infty} \sum_{r=0}^{n-1}\left(\begin{array}{c}
n-1 \\
r
\end{array}\right) \frac{(-1)^{r}}{n^{k}} k(v, u+r) .
$$

Finally, by applying the definition of gamma function on the left hand side of last expression, we easily get our first connection (4.1). Similarly, we can establish our second connection (4.2).

\section{Conclusions}

In the present article, we have proposed two parametric kinds of poly-Bernoulli polynomials by means of two special generating functions. We have also derived some interesting identities for these polynomials by using the series manipulation technique. Furthermore, we have established two connections of our proposed parametric polynomials with the familiar Voigt functions $K(u, v)$ and $L(u, v)$.

\section{References}

[1] T. M. Apostol, On the Lerch zeta function, Pacific J. Math., 1 (1951), 161-167. 1, 1

[2] S. Araci, W. A. Khan, K. S. Nisar, Symmetric identities of Hermite-Bernoulli polynomials and Hermite-Bernoulli numbers attached to a Dirichlet character X, Symmetry, 10 (2018), 10 pages. 1

[3] A. Bayad, Y. Hamahata, Polylogarithms and poly-Bernoulli polynomials, Kyushu J. Math., 65 (2011), 15-24. 1

[4] U. Duran, M. Acikgoz, Truncated Fubini polynomials, Mathematics, 431 (2019), 1-15. 3.8, 3.12

[5] A. Hassen, H. D. Nguyen, Hypergeometric Bernoulli polynomials and Appell sequences, Int. J. Number Theory, 4 (2008), 767-774. 3.14

[6] W. A. Khan, S. Araci, M. Acikgoz, A new class of Laguerre-based Apostol type polynomials, Cogent Math., 3 (2016), 17 pages. $1,3.10,3.11$

[7] W. A. Khan, M. Ghayasuddin, M. Shadab, Multiple-Poly-Bernoulli polynomials of the second kind associated with Hermite polynomials, Fasc. Math., 58 (2017), 97-112.

[8] W. A. Khan, H. Haroon, K. S. Nisar, T. Kim, A new class of generalized Laguerre-based poly-Bernoulli polynomials, Global J. Pure Appl. Math., 13 (2017), 4175-4188.

[9] W. A. Khan, K. S. Nisar, A new class of degenerate Hermite poly-Genocchi polynomials, Preprints, 2018 (2018), 9 pages.

[10] W. A. Khan, K. S. Nisar, Some new classes of generalized Lagrange-Based Apostol type Hermite polynomials, J. Ineq. Special Funct., 10 (2019), 1-11.

[11] W. A. Khan, K. S. Nisar, S. Araci, M. Acikgoz, Fully degenerate Hermite poly Bernoulli numbers and polynomials, Adv. Appl. Math. Sci., 17 (2018), 461-478.

[12] W. A. Khan, K. S. Nisar, U. Duran, M. Acikgoz, S. Araci, Multifarious implicit summation formulae of Hermite-Based poly-Daehee polynomials, Appl. Math. Inf. Sci., 12 (2018), 305-310.

[13] N. U. Khan, T. Usman, J. Choi, Certain generating function of Hermite-Bernoulli-Laguerre polynomials, Far East J. Math. Sci., 101 (2017), 893-908.

[14] N. U. Khan, T. Usman, J. Choi, A new class of generalized polynomials associated with Laguerre and Bernoulli polynomials, Turkish J. Math., 43 (2019), 486-497.

[15] N. U. Khan, T. Usman, K. S. Nisar, A study of generalized Laguerre poly-Genocchi polynomials, Mathematics, 7 (2019), 14 pages.

[16] D. S. Kim, T. Kim, H. Lee, A note on degenerate Euler and Bernoulli polynomials of complex variable, Symmetry, $\mathbf{1 1}$ (2019), 16 pages.

[17] T. Kim, C. S. Ryoo, Some identities for Euler and Bernoulli polynomials and their zeros, Axioms, 7 (2018), 19 pages. 1

[18] T. Komatsu, C. Pita-Ruiz, Truncated Euler polynomials, Math. Slovaca, 68 (2018), 527-536. 3.13

[19] B. Kurt, A further generalization of the Bernoulli polynomials and on the 2D-Bernoulli polynomials $B_{n}^{2}(x, y)$, Appl. Math. Sci. (Ruse), 4 (2010), 2315-2322.

[20] Q.-M. Luo, H. M. Srivastava, Some generalizations of the Apostol-Bernoulli and Apostol-Euler polynomials, J. Math. Anal. Appl., 308 (2005), 290-302. 1

[21] Q.-M. Luo, H. M. Srivastava, Some relationships between the Apostol-Bernoulli and Apostol-Euler polynomials, Comput. Math. Appl., 51 (2006), 631-642. 1

[22] Q.-M. Luo, H. M. Srivastava, q-Extensions of some relationships between the Bernoulli and Euler polynomials, Taiwanese J. Math., 15 (2011), 241-257. 1 
[23] M. A. Pathan, W. A. Khan, Some implicit summation formulas and symmetric identities for the generalized HermiteBernoulli polynomials, Mediterr. J. Math., 12 (2015), 679-695. 1

[24] E. D. Rainville, Special functions, Macmillan Co., New York, (1971). 1

[25] F. Reiche, Uber die emission, absorption and intensitats vertcitung von spektrallicien, Ber. Deutsch. Phys. Ges., 15 (1913), 3-21. 1

[26] H. M. Srivastava, Some formulas for the Bernoulli and Euler polynomials at rational arguments, Math. Proc. Cambridge Philos. Soc., 129 (2000), 77-84. 1

[27] H. M. Srivastava, C. Kizilates, A parametric kind of the Fubini-type polynomials, Rev. R. Acad. Cienc. Exactas Fís. Nat. Ser. A Mat. RACSAM, 113 (2019), 1-15. 1

[28] H. M. Srivastava, H. L. Manocha, A treatise on generating functions, John Wiley \& Sons, New York, (1984). 1

[29] H. M. Srivastava, M. Masjed-Jamei, M. R. Beyki, A parametric type of the Apostol-Bernoulli, Apostol-Euler and ApostolGenocchi, Appl. Math. Inform. Sci., 12 (2018), 907-916. 1 